\title{
Eric Fougère, La Littérature au gré du monde. Espace et réalité de Cervantes à Camus
}

\section{Marta Baravalle}

\section{(2) OpenEdition}

\section{Journals}

Édition électronique

URL : http://journals.openedition.org/studifrancesi/672

DOI : 10.4000/studifrancesi.672

ISSN : 2427-5856

Éditeur

Rosenberg \& Sellier

\section{Édition imprimée}

Date de publication : 1 avril 2015

Pagination : $211-212$

ISSN : 0039-2944

\section{Référence électronique}

Marta Baravalle, "Eric Fougère, La Littérature au gré du monde. Espace et réalité de Cervantes à Camus », Studi Francesi [En ligne], 175 (LIX | I) | 2015, mis en ligne le 01 avril 2015, consulté le 18 septembre 2020. URL : http://journals.openedition.org/studifrancesi/672 ; DOI : https://doi.org/10.4000/ studifrancesi.672

Ce document a été généré automatiquement le 18 septembre 2020

\section{(c) (†) $\odot$}

Studi Francesi è distribuita con Licenza Creative Commons Attribuzione - Non commerciale - Non opere derivate 4.0 Internazionale. 


\title{
Eric Fougère, La Littérature au gré du monde. Espace et réalité de Cervantes à Camus
}

\author{
Marta Baravalle
}

\section{RÉFÉRENCE}

ERIC FOUGÈRE, La Littérature au gré du monde. Espace et réalité de Cervantes à Camus, Paris, Classiques Garnier, 2011, série «Géographies du monde», vol. 17, pp. 230.

1 L'auteur introduit son étude (pp. 7-23) en examinant la qualité du rapport entre l'espace et le réel. À travers une démarche à la fois viatique et panoramique, la thèse d' Éric Fougère s'appuie sur un choix de morceaux littéraires servant à l'analyse du contenu de l'espace, ceci considéré en étant ce qui constitue la possibilité d'apparition du réel. Au lieu d'employer une approche ontologique, l'auteur préfère ainsi mener une enquête sur l'existence, la situation du réel dans l'espace. D'ailleurs, la littérature n'étant pas un art mimétique, elle sous-entendra une représentation, une distance entre l'objet et son écriture, ce qui est la condition d'élection pour interroger le réel. Le choix des extraits, situés principalement entre le $\mathrm{XVII}^{\mathrm{e}}$ et le $\mathrm{xx}^{\mathrm{e}}$ siècle, est fonctionnel au traitement des thèmes concernés par l'auteur, qui avait déjà publié séparément certains chapitres et qui en propose une lecture orientée.

2 Pour ce qui concerne la structure du texte, elle est conçue selon deux configurations visant à souligner la dimension symboliquement ouverte du regard tout court. Dans certains chapitres, l'espace y est projection d'un ordre et le réel un langage, tandis que dans d'autres ce sera plutôt le référent à être mis en question. À ces configurations suivent d'autres répartitions qui de manière systématique mais non exhaustive aspirent à regrouper le matériel présenté selon les différentes caractéristiques des espaces (par ex.: pleins et clos/ouverts et fuyants). Or, le procédé est constitué par une suite de binômes qui enchaîneraient une ouverture de l'horizon critique abordé. 
3 L'art visé par l'auteur est l'instrument qui permet de déceler le dédoublement du regard qui donne sens: partie intégrante du monde qu'il regarde, l'être humain qui se rapport à une réalité livresque peut ainsi prendre conscience de sa vérité.

Pour ce qui est de l'organisation des chapitres, à l'«Introduction» suit un chapitre dédié au chef d'œuvre de Cervantes; ensuite, le livre est reparti en deux sections titrées «Des lieux pour dire. Écrire la terre» et «Des yeux pour voir. Courir le monde», composées respectivement de cinq et quatre chapitres chacune. Une dernière partie est composée d'une conclusion, d'une liste des titres originaux des chapitres déjà parus (p. 211), d'une «Bibliographie» repartie entre primaire et secondaire (pp. 213-217), d'un «Index nominum» (pp. 219-222) et d'un «Index locurom» (pp. 223-225).

Dans Le monde expliqué par la prison: Don Quichotte et la littérature (pp. 25-43), les (més)aventures du célèbre couple espagnol fournissent le prétexte fictif à la création d'un espace romanesque. Dans une double intelligibilité, géographie et littérature se soudent dans la torsion d'une histoire qui s'accomplit dans la traversée d'un espace où l'errance est lecture et quête de gloire. Par la suite, la captivité de Cervantes suggère l'image de la prison comme un monde dépourvu d'enchantement qui démystifie les illusions de la fiction chevaleresque.

6 Le monde en creux: représentation du souterrain romanesque aux XVII et XVIII siècles (pp. 47-59) ouvre la première partie. Ce chapitre explore le symbole de la caverne à travers l'antinomie sous-jacente entre la profondeur close (berceau) et la surface étendue (dévoilement) du Criticón de Baltasar Gracián, pour en venir à l'espace du souterrain comme représentation allégorique de l'esprit, où les antres prennent les semblances du labyrinthe dans Simplicissimus de Grimmelshausen. Lorsque, au XVIII siècle la caverne devient le royaume du renversement de la loi, dans son Robinson Crusoe Defoe en fait ressortir d'espaces nouveaux: elle devient matrice et foyer.

Dans le deuxième chapitre de cette première partie, Le monde en pièces détachées dans "Les caractères" de La Bruyère: écriture de l'espace, espace de l'écriture (pp. 61-75), l'auteur tire profit du recueil de fragments isomorphes mais homogènes de La Bruyère pour opérer une réflexion anthropologique sur l'espace de la ville et notamment sur la frontière, démarcation entre ordre et déraison. Les hommes y sont en effet des «caractères» qui jouent sur la scène de la cour, trait d'union qui rappelle l'existence d'une matrice sociale commune, la mode.

8 En sortant du réel, Le monde en question: les îles oubliées du tournant des Lumières (pp. 77-90) offre un aperçu sur les sociétés utopiques imaginées par François Lefebvre, Claude Gilbert et Pezron de Lesconvel, représentations allégoriques qui reflètent le climat de crise de la conscience européenne au XviII ${ }^{e}$ siècle. Au lieu se substitue le nonlieu et l'île définit ses espaces et ses limites de signification, en abdiquant à une confrontation avec le réel.

Dans Le monde urbanisé de la ville ou le Paris de Louis Sébastien Mercier (pp. 91-104), Éric Fougère reprend ensuite certains concepts de La Bruyère pour en souligner les divergences avec la perspective de l'étude de Mercier. La ville parisienne est réticulaire et en expansion, les caractères appartiennent désormais à différentes classes sociales et ne partagent plus les mêmes lieux. Les frontières deviennent floues, mais les modes permettent un classement qui ne varie que dans la limite opposée par la fixité de la structure cernée par Mercier. 
10 En concluant la première partie du livre, "La ville des expiations" de Pierre-Simon Ballanche, ou la loi des pénitences (pp. 105-116) explore l'eschatologie théosophique du philosophe du $\mathrm{XIX}^{\mathrm{e}}$ siècle. Cette utopie mystique et sociale est réglée par une doctrine expiatoire qui comporte entre autres le déplacement de la question de l'espace dans le temps. La ville des expiations, image chrétienne, serait donc fondamentalement la révélation d'une promesse accomplie.

11 La nature à l'épreuve des îles, ou la grotte et le fraisier de Bernardin de Saint-Pierre (pp. 119-132) entame la seconde partie et marque l'abordage aux thèmes du voyage et du paysage. Selon l'auteur, le botaniste fut le premier à ouvrir la réflexion sur le sentiment de la nature à une esthétique et une philosophie de l'espace. Un système de correspondances y fait appel à une idée d'unité (et de signification) harmonieuse qui relie chaque élément, notamment végétal, à la totalité d'une vision idéalisant la terre.

L'explorateur protagoniste du chapitre titré Une traversée du désert avec René Caillié (pp. 133-149) est chargé par la Société de Géographie d'une mission de recensement dans Tombouctou. Son voyage est culturel, sociologique et anthropologique et représente une des premières incursions européennes dans le continent africain. L'entreprise à caractère coloniale s'enrichit d'une structure à double palimpseste: l'écriture du désert, signe du vide, se dissimule sur les pages d'un Coran, à l'abri des regards ennemis aux chrétiens, et cèle l'espace du voyage relaté.

13 Le Sève et le sang: trajectoire et révolutions de Louise Michel en Nouvelle-Calédonie (pp. 151-163) traite la déportation de la militante communarde, voyage suivi par sa conversion à l'anarchisme. Il s'agit ici d'une expérience existentielle pendant laquelle Louise Michel perçoit dans l'action cyclique du Temps l'inéluctabilité d'une prophétie universelle. La révolution devient ainsi pour l'auteur une potentialité qui se nourrit de l'impossibilité à accomplir les buts proposés.

14 Le désert et la mer sont au centre d'Albert Camus: la mer au plus près, la terre au plus loin (pp. 165-181). En effet, dans La mort heureuse, les personnages du roman observent un horizon tantôt aride tantôt marin, mais cette variété ne serait plus telle pour le lecteur, parce qu'il parvient à y percevoir une signification plus profonde. La pensée sur le monde naît à partir d'une constatation des différentes formes de l'espace, qui dialoguent sur et dans le cycle éternel de la vie et de la mort.

15 L'auteur mène à terme son étude avec Le réel est-il (encore) possible? (pp. 183-209), conclusion dans laquelle il discute l'actualité de la thématique de la terre dans la littérature contemporaine. Pour Éric Fougère, l'évidence demeure un mystère car, il l'a déjà affirmé, l'écriture romanesque n'est pas un art mimétique et elle n'est pas capable de déceler le monde. Lire servira plutôt à prendre conscience de l'absence coupant la continuité d'une pensée qui se veut unique sans s'apercevoir d'être partie d'un ensemble inintelligible. L'espace, donc, est le résultat d'une action qui soude regard et réalité. 